\title{
MULTIPLE HARD X-RAY BURSTS AND ASSOCIATED EMISSIONS
}

\author{
J. VOR PAHL \\ Sacramento City College, Sacramento, Calif., U.S.A.
}

Multiple hard X-radiation, along with associated emission at optical and radio wavelengths, is discussed for three events in particular: December 13, 1970-1832 UT; December 12, 1970-1843 UT; June 28, 1970-2001 UT. Characteristics of these events as well as other multiple hard X-ray bursts observed by the author (e.g., Vorpahl, J. : Solar Phys. 29, 447, 1973) are given at the end. Data originated from hard X-ray experiments on the OGO-5 (Kinsey Anderson) and OSO-5 (Ken Frost) satellites and were compared so that the effect of pulse pile-up in the OGO-5 data could be observed. The two December flares occurred in different active regions separated by about $100000 \mathrm{~km}$ yet both produced hard X-ray bursts with similar structure. This suggests that the two regions were joined by extended field lines with the acceleration mechanism somewhere in between. Although nothing was visible in $\mathrm{H} \alpha$ (such as surges or post flare ejections) that would indicate connecting field lines, higher coronal structures could explain the similarity in hard X-rays from two separate regions.

DECEMBER 3, 1970-1832 UT

\section{$H \alpha$ Characteristics}

(1) $\mathrm{H} \alpha$ kernals were very abrupt and intense, exhibiting secondary enhancements at the time of the first two small $\mathrm{X}$-ray spikes in the $20-32 \mathrm{keV}$ range. The film was too saturated by the time of the third spike to register any increase.

(2) Kernals were directly over good size sunspots of opposite polarity. The spots were growing very fast in a new flux region that had appeared near older spots.

(3) A small filament passed directly through the space between the opposite polarity spots and into one of them.

(4) Only a small amount of material ejection was visible in $\mathrm{H} \alpha$ concurrent with the radio emission at metric wavelengths.

(5) Although very intense, the flare was small in area and of very short duration, lasting only about $12 \mathrm{~min}$ before fading.

\section{Hard X-Ray Burst}

Fairly large with at least three individual spikes as high as $111 \mathrm{keV}$.

\section{Microwave}

Medium size burst with 49 flux units at $8800 \mathrm{MHz}$ and 36 flux units at $15400 \mathrm{MHz}$. 


\section{Long Radio Wavelengths}

Intense type II and III, with weak type IV.

DECEMBER 12, 1970-1843 UT

\section{$H \propto$ Characteristics}

(1) Multiple X-ray spikes corresponded to an optical brightening initially at one end of a big filament, followed by secondary enhancements at both ends of the same filament simultaneous with the secondary hard X-ray spikes.

(2) As in the 12/13/70 event, the flare originated at the site of a new magnetic region growing near some older spots.

(3) Also similar to the $12 / 13 / 70$ flare, the large filament involved in the kernal brightenings was rooted in a region of mixed polarities resulting from the newly emerging flux.

\section{Hard X-Ray Burst}

Fair size, although both the maximum intensity and the highest energy attained $(82 \mathrm{keV})$ were less than the $12 / 13 / 70$ flare.

\section{Microwave}

Event twice as large as that a day later - 115 flux units at $8800 \mathrm{MHz}$ and 22 flux units at $15400 \mathrm{MHz}$.

\section{Long Radio Wavelengths}

Short duration type III bursts of medium intensity. No type II or type IV emission recorded.

JUNE 28, 1970-2001 UT

\section{$H \propto$ Characteristics}

(1) Several $\mathrm{H} \alpha$ kernels coinciding with the initial hard $\mathrm{X}$-ray spike, however, the time resolution on the film was $10 \mathrm{~s}$ and therefore not good enough in this case to know whether the brightening of the many knots really was simultaneous.

(2) The various $\mathrm{H} \alpha$ kernels were in opposite polarities but due to the complexity of the event, it was impossible to identify which pairs were connected by the same flux tube.

(3) Gas ejections were visible in $\mathrm{H} \alpha$ during the approximate time of type II, III, and IV radio emission, but again the event was too complex to identify the optical and long wavelength radio component unambiguously.

(4) The eruption and apparent untwisting of a small filment was observed concurrent with the radio emissions discussed in \#3. 


\section{Hard X-Ray Burst}

Large burst with energies up to $141 \mathrm{keV}$ observed. This event is an example of an event with type IV-like hard X-ray emission described by Ken Frost in these proceedings.

\section{Microwave}

Intense burst with 820 flux units at $8800 \mathrm{MHz}$ and 435 flux units at $15400 \mathrm{MHz}$; Major spikes in frequencies above $2695 \mathrm{MHz}$ tracked the hard X-ray profile.

\section{Long Radio Wavelengths}

Intense type II, III, and IV bursts, with major components in the decimetric type IV mirroring the hard X-rays.

\section{General Characteristics of Multiple X-Ray Bursts}

(1) When multiple hard X-ray bursts occur, there are simultaneous enhancements in the $\mathrm{H} \alpha$ kernel if the film has not already been saturated. Problems in over-exposure are important usually after the second or third component in a multiple burst.

(2) The $\mathrm{H} \alpha$ flare need not be large although the kernels are always intense and brighten abruptly, i.e. within about $20 \mathrm{~s}$.

(3) Ejections in $\mathrm{H} \alpha$ are not necessary but are often observed at the time of III and II or IV events. On the other hand, this author feels that it is still not possible to determine whether there is type III (or type II/IV) flux strictly from observing the $\mathrm{H} \alpha$ flare.

(4) There seems to be a tendency for the microwave and hard X-ray bursts to be more intense when the simultaneous type III is weaker, and vice versa. This suggests either that: (1) there is a preferential beaming of non-thermal electrons up or down; or (2) the magnetic field lines near the acceleration site determine whether any electrons escape upwards, to produce the long wavelength radio emissions or downwards to explain the hard X-ray and microwave flux. 